MODELING, IDENTIFICATION AND CONTROL, 1983, vOL. 4, NO. 2, 83-93

doi:10.4173/mic.1983.22

\title{
Multivariable controller for discrete stochastic amplitude-constrained systems $\dagger$
}

\section{H. T. TOIVONEN}

Keywords: control non-linearities, discrete-time systems, non-linear systems, optimal control, saturation, stochastic control.

\begin{abstract}
A sub-optimal multivariable controller for discrete stochastic amplitudeconstrained systems is presented. In the approach the regulator structure is restricted to the class of linear saturated feedback laws. The stationary covariances of the controlled system are evaluated by approximating the stationary probability distribution of the state by a gaussian distribution. An algorithm for minimizing a quadratic loss function is given, and examples are presented to illustrate the performance of the sub-optimal controller.
\end{abstract}

\section{Introduction}

Optimal control of stochastic systems with amplitude constraints on the inputs has been considered for continuous-time systems (Wonham and Cashman 1969) and for discrete-time single-input systems (Toivonen 1981, $1983 \mathrm{a}, \mathrm{b}$ ). In the discrete-time case an explicit solution to the optimal amplitude-constrained control problem has been determined for first-order systems when the criterion for control is minimum variance of the state. In general the optimal strategy is, however, non-linear and the solution is beyond feasible computation (Fuller 1970). A number of sub-optimal controllers have therefore been designed. Toivonen (1983 a) derives a sub-optimal amplitude-constrained controller for discrete-time single-input systems by approximating the expected future loss in the Bellman equation by a truncated power series including second-order terms. This approach also gives a performance limit in amplitudeconstrained control. Wonham and Cashman (1969) have designed a sub-optimal controller for continuous-time systems by fixing the regulator structure to the class of linear feedback laws with saturation. Toivonen (1983 b) has applied this approach to discrete-time single-input systems. It was found that the approach may give nearoptimal control performance. Successful design of stabilizing saturated linear controllers for deterministic systems has also been reported (Gutman 1982, Gutman and Hagander 1982).

In this paper the procedure described by Toivonen (1983 b) is generalized to multiinput systems. In this method a linear feedback law with saturation is assumed and the covariances of the closed-loop system are evaluated by approximating the stationary probability distribution of the state by a gaussian distribution. Minimization of a stationary quadratic loss function then gives a set of coupled matrix equations which can be solved iteratively to give the optimally tuned feedback gain. The control problem is closely related to the problem of designing optimal structure-constrained

Received 5 January 1983.

† This work was done during the author's stay at the Norwegian Institute of Technology, Division of Engineering Cybernetics, Trondheim, Norway.

$\ddagger$ Department of Chemical Engineering, Åbo Akademi, SF-20500 Turku ( ̊̊bo), Finland. 
controllers (O'Reilly 1980). It is thus straightforward to include the case where only part of the state vector is used for feedback, and this problem is also considered here.

The paper is organized as follows. In $\S 2$ the problem is formulated, and the suboptimal controller is given in $\S 3$. In $\S 4$ numerical examples including an industrial quality control system given in the literature are presented.

\section{Problem formulation}

Consider a discrete-time stochastic system described by

$$
x(t+1)=A x(t)+B u(t)+w(t)
$$

where $\boldsymbol{x}$ is the $\boldsymbol{n}$-dimensional state vector, $\boldsymbol{u}$ is the $m$-dimensional input vector, and $\{\boldsymbol{w}(\boldsymbol{t})\}$ is a sequence of independent gaussian vectors with zero mean value and covariance $R_{w}=E w(t) w(t)^{T}$. It is assumed that the matrix $A$ has all eigenvalues in the unit disc.

We consider the quadratic loss function

$$
J=\underset{N \rightarrow \infty}{E} \lim _{N} \frac{1}{N} \sum_{t=0}^{N-1} x(t)^{T} Q_{1} x(t)+u(t)^{T} Q_{2} u(t)
$$

where $Q_{1}$ and $Q_{2}$ are positive semi-definite weighting matrices. The admissible control signals are assumed to satisfy the amplitude constraints

$$
\left|u_{i}(t)\right| \leqslant \alpha_{i}, \quad i=1, \ldots, m
$$

The control strategy which minimizes the loss function (2.2) subject to the constraints (2.3) is in general non-linear and too complicated to be synthesized readily (Fuller 1970). A sub-optimal regulator can, however, be determined by considering linear saturated feedback laws, i.e.

where

$$
u_{i}(t)=\operatorname{sat}\left(f_{i}^{T} z(t) ; \alpha_{i}\right), \quad i=1, \ldots, m
$$

$$
\text { sat }(\xi ; \alpha)= \begin{cases}\xi, & \text { if }|\xi| \leqslant \alpha \\ \alpha \operatorname{sgn}(\xi), & \text { if }|\xi|>\alpha\end{cases}
$$

and

$$
z(t)=D x(t)
$$

The signal $z(t)$ is introduced in order to study various controller structures where only part of the state vector is used for feedback.

\section{Sub-optimal amplitude-constrained control}

A procedure for tuning the feedback gains in the control law given by eqn. (2.4) to minimize the loss function (2.2) is now considered. Assume that the closed-loop system (2.1), (2.4) is stable. Then the stationary covariances

$$
\left.\begin{array}{l}
R_{x}=E x(t) x(t)^{T} \\
R_{u}=E u(t) u(t)^{T}
\end{array}\right\}
$$

exist and the loss function (2.2) can be written

$$
J=\operatorname{tr} Q_{1} R_{x}+\operatorname{tr} Q_{2} R_{u}
$$


The system eqn. (2.1) gives for the stationary covariances of the closed-loop system the relation

$$
R_{x}=A R_{x} A^{T}+B R_{u x} A^{T}+A R_{u x}{ }^{T} B^{T}+B R_{u} B^{T}+R_{w}
$$

where $R_{u x}=E u(t) x(t)^{T}$. The evaluation of $R_{u x}$ and $R_{u}$ for the saturated feedback (2.4) requires knowledge of the stationary probability distribution of the state, which is non-gaussian due to the non-linear feedback. It is therefore a prohibitively complex task to determine the exact solution of eqn. (3.3) for an amplitude-constrained system. The closed-loop covariances can, however, be determined approximately by using a gaussian distribution to approximate the stationary probability distribution of the state. This approach has been applied successfully by Wonham and Cashman (1969) to the amplitude-constrained control of continuous-time systems, and by Toivonen (1983 b) for discrete-time single-input systems. Mäkilä (1982 a) considers a first-order amplitude-constrained system, showing that for this simple example the probability distribution of the state is reasonably close to a gaussian distribution.

We thus assume for the state $\boldsymbol{x}$ a gaussian probability density function with zero mean value and covariance matrix $R_{x}$ :

$$
p(x)=(2 \pi)^{-n / 2}\left(\operatorname{det} R_{x}\right)^{-1 / 2} \exp \left(-\frac{1}{2} x^{T} R_{x}^{-1} x\right)
$$

Introduce the variances

$$
\sigma_{i}^{2}=f_{i}^{T} R_{z} f_{i}=f_{i}^{T} D R_{x} D^{T} f_{i}, \quad i=1, \ldots, m
$$

Due to (3.4) and (2.4) we have (Toivonen 1983 b)

and

$$
\begin{aligned}
R_{u_{i} x} & =\int_{R^{m}} u_{i}(t) x(t)^{T} p(x) d x \\
& =g_{1 i}\left(\sigma_{i}\right) f_{i}^{T} D R_{x}, \quad i=1, \ldots, m
\end{aligned}
$$

$$
\begin{aligned}
R_{u_{i}} & =\int_{R^{n}} u_{i}(t)^{2} p(x) d x \\
& =g_{2 i}\left(\sigma_{i}\right)^{2} f_{i}^{T} D R_{x} D^{T} f_{i}, \quad i=1, \ldots, m
\end{aligned}
$$

where

$$
\begin{aligned}
& g_{1 i}\left(\sigma_{i}\right)=\operatorname{erf}\left(\alpha_{i} \sigma_{i}^{-1} 2^{-1 / 2}\right) \\
& g_{2 i}\left(\sigma_{i}\right)=\left[\operatorname{erf}\left(\alpha_{i} \sigma_{i}^{-1} 2^{-1 / 2}\right)-\alpha_{i} \sigma_{i}^{-1} 2^{1 / 2} \operatorname{ierfc}\left(\alpha_{i} \sigma_{i}^{-1} 2^{-1 / 2}\right)\right]^{1 / 2}
\end{aligned}
$$

Introducing the feedback matrix

and the diagonal matrices

$$
F=\left[f_{i}^{T}\right]
$$

we have

$$
\begin{aligned}
& G_{1}=\operatorname{diag}\left(g_{1 i}\left(\sigma_{i}\right)\right) \\
& G_{2}=\operatorname{diag}\left(g_{2 i}\left(\sigma_{i}\right)\right)
\end{aligned}
$$

$$
R_{u x}=G_{1} F D R_{x}
$$

and, introducing the approximation that the stochastic variables $u_{i}, u_{j}$, eqn. (2.4), have the same correlation coefficient as the normally distributed variables $f_{i}{ }^{T} z, f_{j}{ }^{T} z$, gives the approximate relation

$$
R_{u}=G_{2} F D R_{x} D^{T} F^{T} G_{2}{ }^{T}
$$

The expressions (3.10) and (3.11) can be introduced into eqn. (3.3), which can be solved iteratively to give approximate values for the stationary covariances of the closed-loop system. Numerical examples show $(\S 4)$, that in spite of the approximations 
involved the procedure gives estimates of the closed-loop covariances which are typically within $10 \%$ of the true values.

Now consider the problem of tuning the feedback matrix $F$ to minimize the loss function $J$. Equations (3.2) and (3.11) give

$$
J(F)=\operatorname{tr}\left(Q_{1}+D^{T} F^{T} G_{2}{ }^{T} Q_{2} G_{2} F D\right) R_{x}
$$

Using eqns. (3.3) and (3.8) to (3.11) some rather tedious but fairly straightforward matrix manipulations give the gradient of $J$ with respect to $F$ :

$$
\begin{aligned}
& \frac{\partial J}{\partial F}=2\left[G_{1}{ }^{T} B^{T} S A+G_{2}{ }^{T}\left(B^{T} S B+Q_{2}\right) G_{2} F D\right] R_{x} D^{T} \\
& +4 \sum_{l=1}^{m} E_{l l}\left[h_{1 l}\left(\sigma_{l}\right) B^{T} S A+h_{2 l}\left(\sigma_{l}\right)\left(B^{T} S B+Q_{2}\right) G_{2} F D\right] \\
& \times R_{x} D^{T} F^{T} E_{l l} F D R_{x} D^{T}
\end{aligned}
$$

where $S$ is the symmetric positive definite solution to

$$
\begin{aligned}
& S=A^{T} S A+A^{T} S B G_{1} F D+D^{T} F^{T} G_{1}{ }^{T} B^{T} S A \\
& +D^{T} F^{T} G_{2}{ }^{T}\left(B^{T} S B+Q_{2}\right) G_{2} F D+Q_{1}+2 D^{T} F^{T} \sum_{l=1}^{m} E_{l l}\left[h_{1 l}\left(\sigma_{l}\right) B^{T} S A\right. \\
& \left.+h_{2 l}\left(\sigma_{l}\right)\left(B^{T} S B+Q_{2}\right) G_{2} F D\right] R_{x} D^{T} F^{T} E_{l l} F D
\end{aligned}
$$

and

$$
\begin{aligned}
& h_{1 l}\left(\sigma_{l}\right)=\frac{\partial g_{1 l}\left(\sigma_{l}\right)}{\partial \sigma_{l}{ }^{2}}=-(2 \pi)^{-1 / 2} \alpha_{l} \sigma_{l}{ }^{-3} \exp \left(-\frac{1}{2} \alpha_{l}{ }^{2} \sigma_{l}{ }^{-2}\right) \\
& h_{2 l}\left(\sigma_{l}\right)=\frac{\partial g_{2 l}\left(\sigma_{l}\right)}{\partial \sigma_{l}{ }^{2}}=-\frac{1}{2} \alpha_{l}{ }^{2} \sigma_{l}{ }^{-4} g_{2 l}\left(\sigma_{l}\right)^{-1} \operatorname{erfc}\left(\alpha_{l} \sigma_{l}{ }^{-1} 2^{-1 / 2}\right)
\end{aligned}
$$

The matrix $E_{l l}$ is an $m \times m$ matrix with zeros everywhere except for the element $l l$, which is unity.

The minimum of $J(F)$ can be found by applying function minimization techniques. Alternatively various special-purpose methods can be developed. Here we consider a linear descent algorithm, which is related to a procedure used in the design of optimal structure-constrained controllers (O'Reilly 1980, Polak 1971, Mäkilä 1982 b). A similar algorithm has previously been applied for computing sub-optimal amplitudeconstrained controllers for single-input systems (Toivonen $1983 \mathrm{~b}$ ). The algorithm solves the necessary optimality condition $\partial J / \partial F=0$ iteratively, generating a sequence $\left\{F_{k}\right\}$ of feedback gains according to

$$
F_{k+1}=F_{k}+a_{k} T_{k}
$$

where $a_{k}$ is a steplength parameter and

$$
\begin{aligned}
& T_{k}=-\left[G_{2}{ }^{(k) T}\left(B^{T} S^{(k)} B+Q_{2}\right) G_{2}{ }^{(k)}\right]^{-1}\left[G_{1}{ }^{(k) T} B^{T} S^{(k)} A R_{x}{ }^{(k)} D^{T}\left(D R_{x}{ }^{(k)} D^{T}\right)^{-1}\right. \\
& +2 \sum_{i=1}^{m} E_{l l}\left[h_{1 l}\left(\sigma_{t}{ }^{(k)}\right) B^{T} S^{(k)} A+h_{2 l}\left(\sigma_{t}{ }^{(k)}\right)\left(B^{T} S^{(k)} B\right.\right. \\
& \left.\left.\left.+Q_{2}\right) G_{2}{ }^{(k)} F_{k} D\right] R_{x}{ }^{(k)} D^{T} F_{k}{ }^{T} E_{l l} F_{k}\right]-F_{k} \\
& =-\frac{1}{2}\left[G_{2}{ }^{(k) T}\left(B^{T} S^{(k)} B+Q_{2}\right) G_{2}{ }^{(k)}\right]^{-1}\left(\frac{\partial J}{\partial F}\right)_{k}\left(D R_{x}{ }^{(k)} D^{T}\right)^{-1}
\end{aligned}
$$


Here $S^{(k)}, R_{x}{ }^{(k)}, \sigma_{l}{ }^{(k)}, G_{1}{ }^{(k)}, G_{2}{ }^{(k)}$ are evaluated for $F_{k}$. The direction $T_{k}$ is a descent direction of the loss function $J$ at $F_{k}$, because

$$
\operatorname{tr}\left(\frac{\partial J}{\partial F}\right)_{k}^{T} T_{k}<0, \quad \text { if }\left(\frac{\partial J}{\partial F}\right)_{k} \neq 0
$$

due to (3.17) and the positive definiteness of the matrices $G_{2}{ }^{T}\left(B^{T} S B+Q_{2}\right) G_{2}$ and $D R_{x} D^{T}$. There thus exists an $a_{m}>0$, such that for all $a_{k} \in\left(0, a_{m}\right)$

$$
J\left(F_{k+1}\right)<J\left(F_{k}\right), \quad \text { if }\left(\frac{\partial J}{\partial F}\right)_{k} \neq 0
$$

By choosing the steplength parameter $a_{k}$ properly (Goldstein 1965, Fletcher 1980), the algorithm gives convergence to a local minimum of the loss, i.e. (Mäkilä 1982 b)

$$
\left(\frac{\partial J}{\partial F}\right)_{k}=0, \text { or }\left(\frac{\partial J}{\partial F}\right)_{k} \rightarrow 0
$$

for any starting point $F_{0}$ for which $J$ is finite.

\section{Examples}

Numerical examples are now given to illustrate the performance of the amplitudeconstrained controller.

\section{Example 1.}

Consider a system described by eqn. (2.1) with the parameters

$$
A=\left[\begin{array}{cc}
1 & 0.1 \\
-0.5 & 0.9
\end{array}\right], \quad B=\left[\begin{array}{cc}
1 & -0.4 \\
-0.8 & 0.9
\end{array}\right], \quad R_{w}=\left[\begin{array}{ll}
1 & 0 \\
0 & 1
\end{array}\right]
$$

and the loss function (2.2) with $Q_{1}=I, Q_{2}=0 \cdot 11$. The optimal unconstrained strategy is

where

$$
u(t)=F^{*} x(t)
$$

$$
F^{*}=\left[\begin{array}{cc}
-0.932 & -0.383 \\
-0.198 & -1 \cdot 153
\end{array}\right]
$$

giving the loss $J=2 \cdot 347$. The input variances in the unconstrained case are $r_{u_{1}}=1 \cdot 10$ and $r_{u_{2}}=1.44$.

Sub-optimal amplitude-constrained controllers were determined using the procedure described in $\S 3$. The results are summarized in Tables 1 and 2.

For comparison, the performance of the 'standard' control, obtained by using the optimal unconstrained feedback gain $F^{*}$ in the saturated feedback law (2.4), is also given. As this controller has been designed for the case when the inputs are unconstrained, it does not perform well when there are constraints on the input amplitudes. This is particularly true when the constraints are tight and in the case when the relative magnitudes of the constraints on the various inputs differ (c.f. Table 1). In these cases much can be gained by tuning the feedback gain properly. 


\begin{tabular}{|c|c|c|c|c|c|c|}
\hline \multirow[b]{3}{*}{$\alpha$} & \multicolumn{4}{|c|}{ Sub-optimal control } & \multicolumn{2}{|c|}{ Standard control } \\
\hline & & & & oss & & oss \\
\hline & & & Estimated & Simulated & Estimated & Simulated \\
\hline$\left[\begin{array}{l}1 \\
1 \cdot 5\end{array}\right]$ & {$\left[\begin{array}{r}-0.755 \\
0.165\end{array}\right.$} & $\left.\begin{array}{l}-0.240 \\
-0.979\end{array}\right]$ & $2 \cdot 65$ & $2 \cdot 69$ & $2 \cdot 82$ & $2 \cdot 96$ \\
\hline$\left[\begin{array}{l}1 \\
1\end{array}\right]$ & {$\left[\begin{array}{r}-0.745 \\
0.143\end{array}\right.$} & $\left.\begin{array}{c}-0.062 \\
-0.840\end{array}\right]$ & $2 \cdot 79$ & $2 \cdot 83$ & $3 \cdot 11$ & 3.61 \\
\hline$\left[\begin{array}{l}1 \\
0 \cdot 5\end{array}\right]$ & {$\left[\begin{array}{r}-0.745 \\
0.120\end{array}\right.$} & $\left.\begin{array}{r}0.168 \\
-0.552\end{array}\right]$ & $3 \cdot 14$ & $3 \cdot 17$ & $14 \cdot 33$ & $17 \cdot 27$ \\
\hline$\left[\begin{array}{l}0.5 \\
0.5\end{array}\right]$ & {$\left[\begin{array}{r}-0.590 \\
0.408\end{array}\right.$} & $\left.\begin{array}{r}0.116 \\
-0.426\end{array}\right]$ & $4 \cdot 34$ & $4 \cdot 68$ & $7 \cdot 95$ & $9 \cdot 62$ \\
\hline$\left[\begin{array}{l}0 \cdot 3 \\
0 \cdot 3\end{array}\right]$ & {$\left[\begin{array}{r}-0.611 \\
0.501\end{array}\right.$} & $\left.\begin{array}{r}0.077 \\
-0.247\end{array}\right]$ & $9 \cdot 13$ & $10 \cdot 10$ & $18 \cdot 31$ & $19 \cdot 63$ \\
\hline
\end{tabular}

Table 1. Results for Example 1. Estimated: loss function calculated by approximate procedure using gaussian distributions. Simulated: loss function obtained as a sample average $\left(N=10^{6}\right)$.

The feedback gains of the sub-optimal amplitude-constrained controllers given in Table 1 correspond approximately to the feedback gains obtained by standard linearquadratic-gaussian design, the amplitude constraints corresponding to an increased weighting of the inputs. For example, the linear-quadratic-gaussian controller obtained for $Q_{2}=I$, has the feedback matrix

$$
F=\left[\begin{array}{rr}
-0.537 & 0.040 \\
0.123 & -0.498
\end{array}\right]
$$

which can be compared with the feedback matrices of the amplitude-constrained controllers (Table 1). Inspection of eqns. (3.13) and (3.14) shows, however, that the sub-optimal amplitude-constrained controllers cannot be generated exactly by linear-quadratic-gaussian design for any choice of the input weighting matrix $Q_{2}$.

The accuracy of the approximate procedure for evaluating the closed-loop covariances using gaussian distribution functions is illustrated in the tables by also giving the values of the loss function and the covariances obtained by Monte-Carlo simulation. When the constraints are not too tight $(\alpha>0.5)$, the errors in the covariances are less than $10 \%$.

\section{Example 2}

In this example an industrial quality control system is considered. The example also gives a comparison of the amplitude-constrained controller and the use of linear 


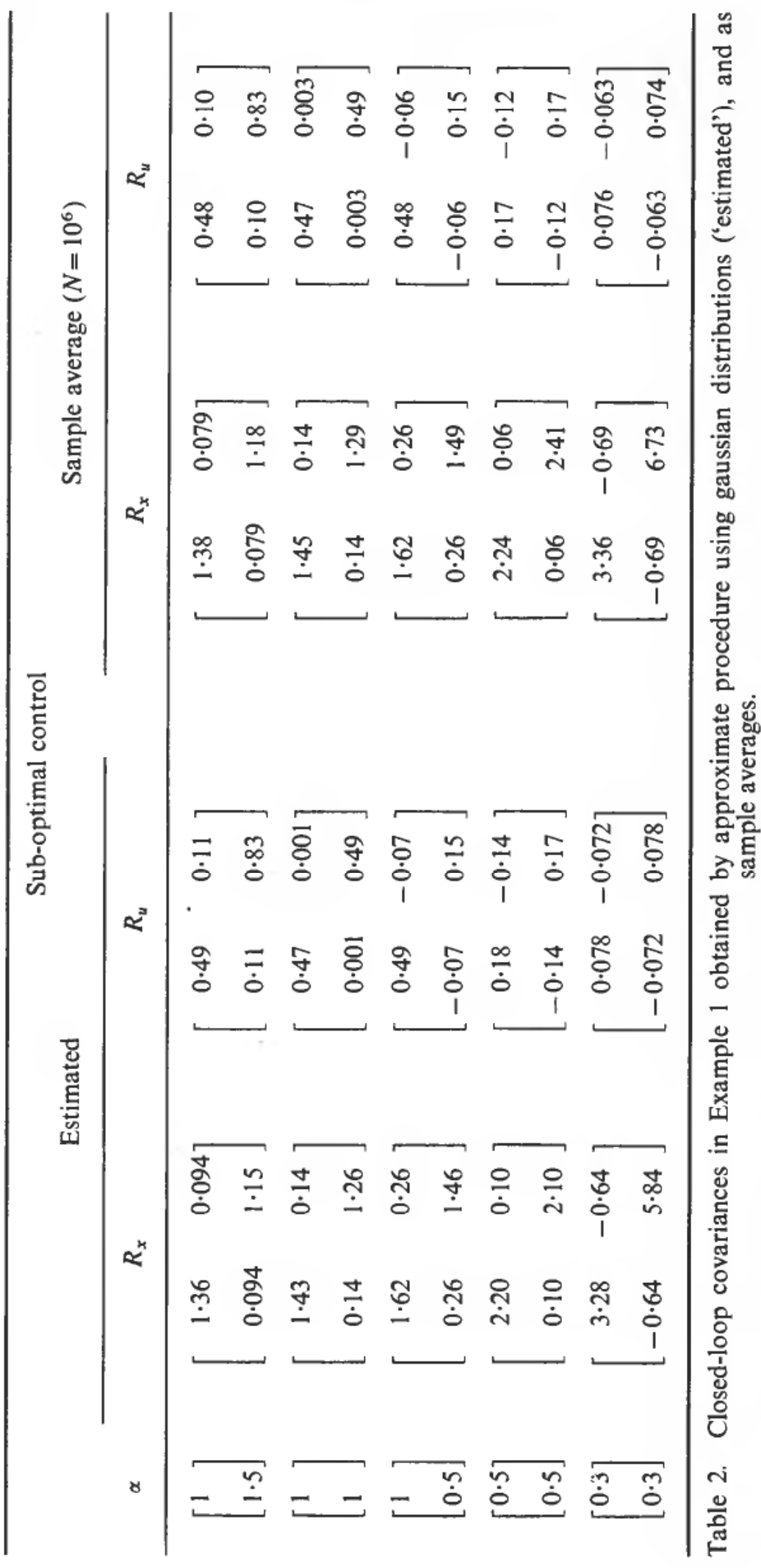


quadratic gaussian design for reducing the input variances. Westerlund (1981) considers the digital control of an industrial cement kiln. A state-space representation of the model obtained by maximum likelihood identification is

$$
\left.\begin{array}{rl}
x(t+1) & =A x(t)+B u(t)+D e(t+1) \\
y(t) & =\left[\begin{array}{lll}
1 & 0 & 0 \\
0 & 1 & 0
\end{array}\right] x(t)
\end{array}\right\}
$$

where

$$
\begin{aligned}
& A=\left[\begin{array}{cll}
0.914 & 0.080 & 0 \\
-0.126 & 0.917 & 0.715 \\
0 & 0 & 0
\end{array}\right], \quad B=\left[\begin{array}{cc}
2.091 & -0.0744 \\
-0.211 & -0.0156 \\
0 & 0
\end{array}\right] \\
& R_{e}=\left[\begin{array}{ll}
0.0644 & 0.000257 \\
0.000257 & 0.0214
\end{array}\right], \quad D=\left[\begin{array}{ll}
1 & 0 \\
0 & 1 \\
0 & 1
\end{array}\right]
\end{aligned}
$$

The loss function was (Westerlund 1981)

$$
J=\operatorname{tr} R_{y}=r_{y_{1}}+r_{y_{2}}
$$

The optimal unconstrained strategy is given by

$$
\boldsymbol{u}(t)=\left[\begin{array}{cc}
-0.490 & 2.49 \\
-1.49 & 71.1
\end{array}\right] \boldsymbol{y}(t)
$$

and the closed-loop variances are then

$$
\begin{array}{ll}
r_{y_{1}}=0.0644, & r_{y_{2}}=0.0214 \\
r_{u_{1}}=0.148, & r_{u_{2}}=108 .
\end{array}
$$

Due to physical constraints in the process the input variances were restricted according to

$$
r_{u_{1}} \leqslant 0 \cdot 004, \quad r_{u_{2}} \leqslant 1.5
$$

Westerlund (1981) applied constrained linear quadratic gaussian design (Mäkilä, Westerlund and Toivonen 1982) to minimize the loss function (4.4) subject to the variance constraints (4.7). The optimal controller consists of a state estimator and a feedback from the estimated state. In order to obtain a simpler controller a feedback from the process output $y(t)$ only was considered. The optimal output controller is given by

$$
u(t)=\left[\begin{array}{rr}
-0.177 & 0.125 \\
1.84 & 2.09
\end{array}\right] y(t)
$$


which gives the closed-loop variances

$$
\begin{array}{ll}
r_{y_{1}}=0.0933, & r_{y_{2}}=0.193 \\
r_{u_{1}}=0.004, & r_{u_{2}}=1.5
\end{array}
$$

and the loss $J=0 \cdot 286$.

Table 3 gives the results obtained with sub-optimal amplitude-constrained controllers. Two feedback structures are considered: (1) feedback from the whole state vector $\hat{\boldsymbol{x}}(t \mid t)$ estimated using a Kalman filter, and (2) feedback from the process output $y(t)$ only, corresponding to the structure of the controller (4.8) used in

\begin{tabular}{|c|c|c|c|c|c|c|c|c|}
\hline \multicolumn{4}{|c|}{$\begin{array}{c}\text { Sub-optimal controller } \\
u_{i}(t)=\operatorname{sat}\left(f_{i}^{T} \hat{x}(t \mid t) ; \alpha_{i}\right)\end{array}$} & \multicolumn{5}{|c|}{$\begin{array}{c}\text { Sub-optimal controller } \\
u_{i}(t)=\operatorname{sat}\left(f_{i}^{T} y(t) ; \alpha_{i}\right)\end{array}$} \\
\hline$\alpha$ & & $\begin{array}{l}\text { Esti- } \\
\text { mated }\end{array}$ & $\begin{array}{l}\text { Simu- } \\
\text { lated }\end{array}$ & $F$ & & & $\begin{array}{l}\text { Esti- } \\
\text { mated }\end{array}$ & $\begin{array}{l}\text { Simu- } \\
\text { lated }\end{array}$ \\
\hline$\left[\begin{array}{l}0 \cdot 25 \\
5 \cdot 0\end{array}\right]$ & $r_{y},:$ & 0.074 & 0.098 & {$\left[\begin{array}{l}-0.553 \\
-1.859\end{array}\right.$} & $\left.\begin{array}{c}0 \cdot 771 \\
41 \cdot 7\end{array}\right]$ & $r_{y_{1}}:$ & 0.074 & 0.099 \\
\hline & $r_{y_{2}}$ & 0.073 & 0.075 & & & $r_{y_{2}}:$ & 0.079 & 0.081 \\
\hline & $r_{u_{1}}:$ & 0.031 & 0.032 & & & $r_{u_{1}}$ & 0.031 & 0.033 \\
\hline & $r_{u_{2}}:$ & $19 \cdot 4$ & $19 \cdot 2$ & & & $r_{u_{2}}:$ & $19 \cdot 4$ & $18 \cdot 8$ \\
\hline & Loss: & $0 \cdot 147$ & $0 \cdot 173$ & & & Loss: & $0 \cdot 153$ & $0 \cdot 180$ \\
\hline$\left[\begin{array}{l}0 \cdot 15 \\
3 \cdot 0\end{array}\right]$ & $r_{y_{1}}:$ & 0.085 & $0 \cdot 102$ & {$\left[\begin{array}{c}-0.485 \\
4.69\end{array}\right.$} & $\left.\begin{array}{c}0 \cdot 508 \\
25 \cdot 3\end{array}\right]$ & $r_{y_{1}}:$ & 0.085 & $0 \cdot 103$ \\
\hline & $r_{y_{2}}:$ & $0 \cdot 122$ & $0 \cdot 127$ & & & $r_{y_{2}}:$ & $0 \cdot 127$ & $0 \cdot 132$ \\
\hline & $r_{u_{1}}:$ & 0.014 & 0.014 & & & $r_{u_{1}}:$ & 0.014 & 0.014 \\
\hline & $r_{u_{2}}$ & $7 \cdot 52$ & $7 \cdot 41$ & & & $r_{u_{2}}:$ & $7 \cdot 49$ & $7 \cdot 29$ \\
\hline & Loss: & 0.207 & 0.229 & & & Loss: & 0.212 & 0.235 \\
\hline
\end{tabular}
Westerlund (1981).

Table 3. Results for Example 2. Estimated: using the approximate procedure of $\S 3$. Simulated: sample average $\left(N=10^{6}\right)$.

The amplitude constraint $\alpha=[0 \cdot 25,5]^{T}$ corresponds to the amplitudes of the PRBS signals used in an $8 \mathrm{~h}$ identification experiment (Westerlund 1981). For comparison, a more restrictive constraint is also considered.

In analogy with the discussion in Example 1, it is seen that the feedback gains of the sub-optimal amplitude-constrained controllers correspond qualitatively to linearquadratic-gaussian controllers with a penalty on the input variances: c.f. eqns. (4.5) and (4.8) and Table 3. 
The Figure shows the inputs and the outputs of the system when using the suboptimal amplitude-constrained strategy. The results show that good control performance is obtained with realistic values for the amplitude constraints. In practice a design procedure which combines amplitude constraints and variance restrictions of the type (4.7) could be useful.

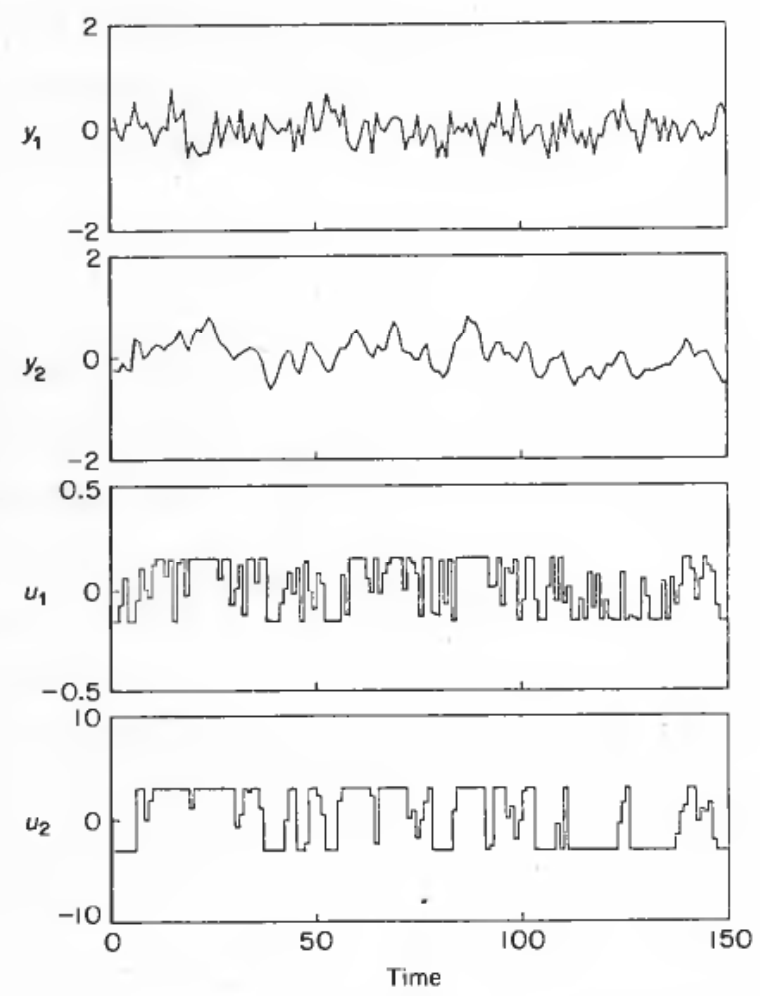

Inputs and outputs of the system (4.3) when controlled by the amplitude-constrained controller $u_{i}=$ sat $\left(f_{i}^{T} y ; \alpha_{i}\right), \alpha=[0 \cdot 15,3]^{T}$, of Table 3 .

\section{Conclusions}

The paper presents a sub-optimal controller for multivariable stochastic systems for the case when the control signals are constrained in amplitude. The structure of the controller is taken to be a linear feedback with saturation. Previous investigations dealing with continuous time systems (Wonham and Cashman 1969) and discrete time single-input systems (Toivonen 1983 b) have shown that linear saturated controllers may give near-optimal control performance.

The procedure described in the paper is a multivariable generalization of the method given in Toivonen (1983 b). In this approach the closed-loop covariances of the amplitude-constrained system are evaluated by approximating the stationary probability distribution of the state by a gaussian distribution. In numerical examples this approach has given sufficiently accurate estimates of the closed-loop covariances to be useful for optimization of the regulator parameters. Minimization of a stationary quadratic loss function gives a set of coupled matrix equations which can be solved iteratively to give the optimal feedback matrix. 
The procedure gives a straightforward and computationally simple design method for amplitude-constrained systems. Examples show that it could be useful in many practical situations. The method is, however, restricted to open-loop stable systems, as an unstable stochastic system cannot in general be stabilized when the inputs are constrained in amplitude.

\section{ACKNOWLEDGMENT}

During this work the author received financial support from The Royal Norwegian Council for Scientific and Industrial Research.

\section{REFERENCES}

Fletcher, R. (1980). Practical Methods of Optimization, Vol. 1, New York: Wiley.

Fuller, A. T. (Ed.) (1970). Nonlinear Stochastic Control Systems, London: Taylor \& Francis. Goldstein, A. A. (1965). On steepest descent, SIAM J. Cont., 3, 147-151.

Gutman, P.-O. (1982). Controllers for bilinear and constrained linear systems. CODEN: LUTFD2/(TFRT-1022)/1-133/(1982), Dep. Aut. Contr., Lund Univ., Sweden.

Gutman, P.-O., and Hagander, P. (1982). A new design of constrained controller for linear systems. Proceedings of the 21st I.E.E.E. Conference on Decision and Control, Florida.

MÄKıLÄ, P. M. (1982 a). Self-tuning control with linear input constraints, Optimal Control Appl. \& Methods, 3, 337-353.

MÄKıLÄ, P. M. (1982 b). Constrained linear quadratic gaussian control for process application. Ph.D. Thesis, Åbo Akademi, Turku, Finland.

MÄKılä, P. M., Westerlund, T., and ToIvonen, H. T. (1982). Constrained linear quadratic gaussian control. Proceedings of the 21st I.E.E.E. Conference on Decision and Control, Florida.

O'Reilly, J. (1980). Optimal low-order feedback controllers for linear discrete-time systems. In Control and Dynamic Systems, edited by C. T. Leondes, New York: Academic Press, Vol. 16, pp. 335-367.

PolaK, E. (1971). Computational Methods in Optimization, New York: Academic Press.

ToIVONEN, H. T. (1981). Minimum variance control of first-order systems with a constraint on the input amplitude, I.E.E.E. Trans. Autom. Control, AC-26, 556-558.

ToIvONEN, H. T. (1983 a). Suboptimal control of linear discrete stochastic systems with linear input constraints, I.E.E.E. Trans. Autom. Control, AC-28, 246-248.

ToIvonen, H. T. (1983 b). Suboptimal control of discrete stochastic amplitude-constrained systems, Int. J. Control, 37, 493-502.

WESTERLUND, T. (1981). A digital quality control system for an industrial dry process rotary cement kiln, I.E.E.E. Trans. Autom. Control, AC-26, 885-890.

Wonham, W. M., and CASHMAN, W. F. (1969). A computational approach to optimal control of stochastic saturating systems, Int. J. Control, 10, 77-98. 